\title{
Imaging At the Timescale Of Micro- and Milliseconds With the pnCCD (S)TEM Camera
}

H. Ryll ${ }^{1}$, M. Simson ${ }^{2}$, M. Den Hertog ${ }^{3}$, R. Dunin-Borkowski ${ }^{4}$, K. El Hajraoui ${ }^{3}$, R. Hartmann ${ }^{1}$, M. Huth ${ }^{1}$, S. Ihle ${ }^{1}$, V. Migunov ${ }^{4}$, J. Schmidt ${ }^{3}$, H. Soltau ${ }^{2}$ and L. Strüder ${ }^{1}$

1. PNSensor GmbH, Otto-Hahn-Ring 6, 81739 Munich, Germany

2. PNDetector GmbH, Otto-Hahn-Ring 6, 81739 Munich, Germany

3. Institut Néel-CNRS, 25 Rue des Martyrs, 38042 Grenoble, France

4. Ernst Ruska-Centre, Peter Grünberg Institute, Forschungszentrum Jülich, Leo-Brandt-Straße, 52425

Juelich, Germany

Dynamic processes that can, in principle, be observed in TEM imaging can be too fast to be resolved with conventional cameras which are typically running below 40 frames per second (fps). The pnCCD (S)TEM camera is routinely running at $1000 \mathrm{fps}$ in full frame mode [1]. This camera uses a direct detecting, radiation hard pnCCD with 264x264 pixels and features binning and windowing modes which substantially increase the frame rate. For example, 4-fold binning in one direction, i.e. 66x264 pixels, yields a readout speed of $4000 \mathrm{fps}$. In windowing modes up to $20000 \mathrm{fps}$ are possible.

The propagation of a metal-semiconductor phase inside a nanowire was observed in-situ with the pnCCD (S)TEM camera. A silicon nanowire was contacted with Pt strips at each end in an electrical biasing holder. By flowing a current through a Pt strip the nanowire's temperature was controlled by Joule heating which excited the propagation of a Pt-Si phase into the nanowire [2]. Images of the nanowire during the growth process were recorded continuously with $1000 \mathrm{fps}$ for over 30 minutes. The resulting high speed movie shows the growth process with millisecond time resolution. Single frames from the movie are shown in Figure 1. Various abrupt propagation steps could be observed in the movie. This shows the potential of the pnCCD (S)TEM camera for imaging of dynamic processes at the millisecond timescale.

The flexible configuration of the camera readout permitted a mode with even shorter integration times down to $25 \mu$ s at a readout speed of 1850 images per second. Such a short integration time was required for imaging of oscillations of a $\mathrm{CdS}$ nanowire. The experimental setup consisted of a free standing nanowire [3] that was brought close to a probe tip. A sine voltage was applied to the probe tip that induced resonant vibrations of the nanowire. Part of the oscillating nanowire's shadow image was then recorded with the camera. The readout of the camera was triggered with a variable delay with respect to the zero crossing of the sine function (see Figure 2a). In this way, it was possible to sample the position of the nanowire at the corresponding value of the sine voltage. The decisive configuration was to decrease the integration time of each single image to $25 \mu \mathrm{s}$, otherwise the high velocity movement of the nanowire would blur the image. The excitation frequency was $1850 \mathrm{~Hz}$, the camera was read out with the same speed. In order to shorten the integration time, only a part of the image area was read out. The readout sequence starting at the trigger signal consisted of three steps: 1) clear pixel of signal charge, 2) integrate for a set time (here $25 \mu \mathrm{s}$ ), 3) readout of charge collected in the pixel.

In the analysis of the measurement, for each delay, the center of gravity of the intensity distribution was calculated for each image as the position of the nanowire. The dependency of the position of the nanowire on the delay and hence the exciting voltage reveals that the resonant frequency of the nanowire 
is approximately 4 times the excitation frequency (Figure 2b). Elastic properties of the nanowire could then be deduced through further analysis.

In conclusion, the imaging capabilities of the pnCCD (S)TEM camera at millisecond time scales and below have been shown. Live, high-speed movies of platinum growth in nanowires were recorded with 1000 frames per second. Integration times of single images down to $25 \mu$ s were possible with flexible readout modes.

[1] H. Ryll et al., Microscopy and Microanalysis 19 (2013), p.1160-1161

[2] M. Mongillo et al., ACS Nano 5 (2011), p. 7117-7123

[3] R. Liu et al., Nano Letters 13 (2013), p. 2997-3001
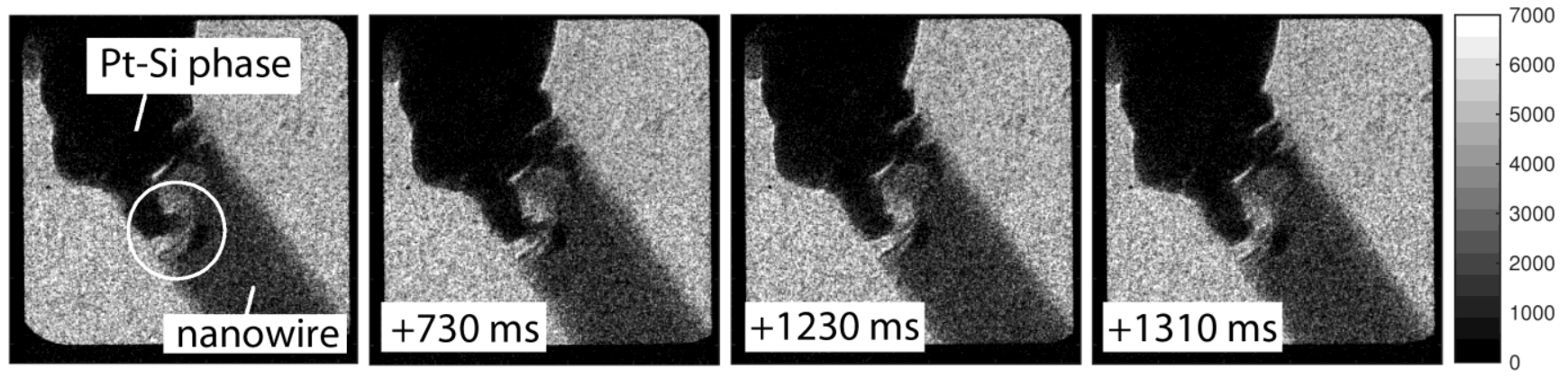

Figure 1. Examples of single raw images from a movie sequence in which a Pt-Si phase is propagating in a silicon nanowire. Notice the area of growth indicated by the circle in the first image.

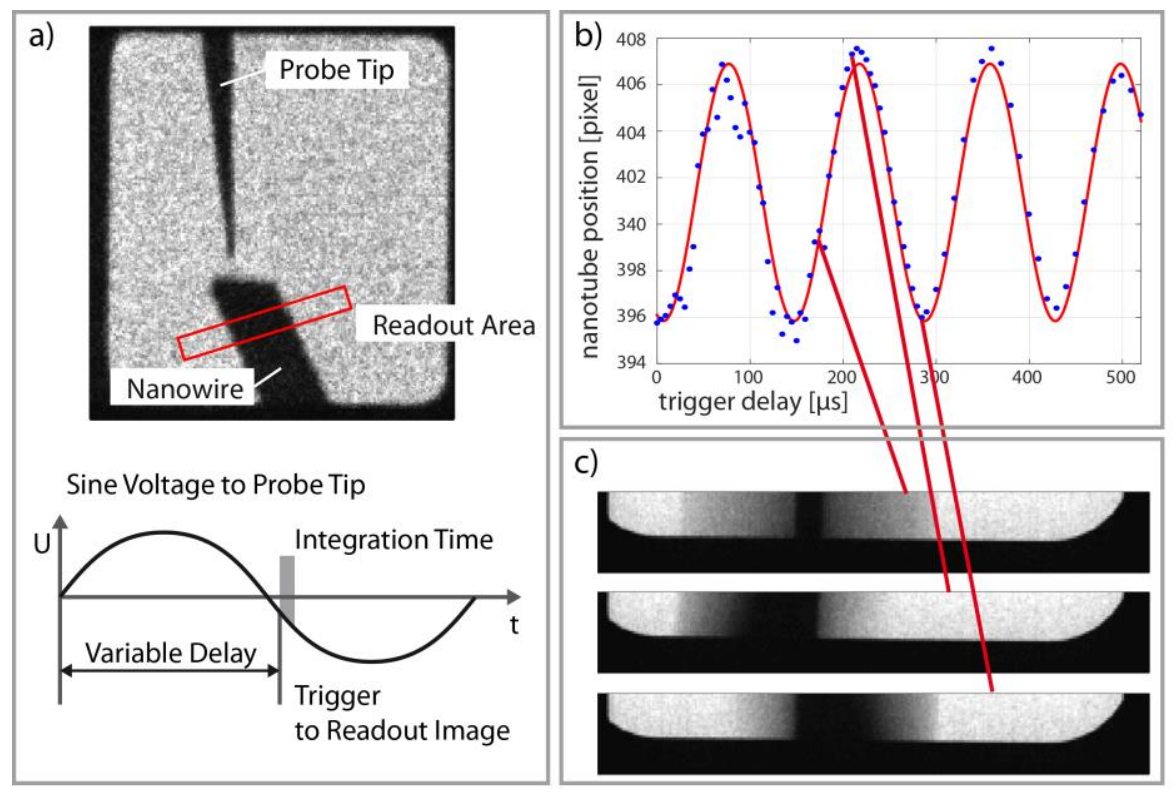

Figure 2. Overview image of nanowire and probe tip position in a). A sine voltage was applied to the probe tip inducing oscillations in the nanowire. The shadow of the vibrating nanowire was imaged with an integration time of $25 \mu \mathrm{s}$. The center of gravity of these images was calculated and plotted against the trigger delay in b). Single images of the readout area are shown in c) for three different delays. 